\title{
LOS COMITÉS DE ÉTICA DE INVESTIGACIÓN BIOMÉDICAY PSICOSOCIAL EN BOLIVIA
}

\begin{abstract}
Elizabeth C. Zwareva Mashiri*
Resumen: Este artículo reporta un estudio realizado en las ciudades de La Paz, Cochabamba y Santa Cruz en Bolivia. Interesaba saber cuál era la situación de la bioética en Bolivia y cómo se estaba protegiendo a los sujetos de investigación.

Luego de revisar algunos conceptos bioéticos, la legislación boliviana y los métodos del estudio se dan a conocer los resultados del mismo, los cuales revelan gran desconocimiento respecto de cómo proteger los derechos de los sujetos, por la casi ausencia o el inadecuado funcionamiento de los comités de ética de la investigación.
\end{abstract}

Palabras clave: sujetos de investigación, comité de ética, principios bioéticos, dignidad humana

\section{BIOMEDICAL AND PSICOSOCIAL SCIENTIFIC ETHICAL REVIEW COMMITTEES IN BOLIVIA}

Abstract: This article reports a study carried out in the cities of La Paz, Cochabamba and Santa Cruz in Bolivia. The interest was to know the situation of bioethics in Bolivia and how research subjects are being protected.

After revising some bioethical concepts, Bolivian legislation and study methods, results revealed great lack of knowledge on how to protect research subjects, due to the almost absence or inadequate functioning of scientific ethical review committees.

Key words: research subjects, ethics committee, bioethics principles, human dignity

\section{OS COMITÊS DE ÉTICA DE PESQUISA BIOMÉDICA E PSICOSSOCIAL NA BOLIVIA}

Resumo: Este artigo reporta a um estudo realizado nas cidades de La Paz, Cochabamba, e Santa Cruz, na Bolivia. Interessava-se saber qual era a situaçáo da bioética na Bolivia e como se estava protegendo os sujeitos de pesquisa. Ao revisar alguns conceitos bioéticos, a legislação boliviana e os métodos de estudo foi possível identificar os resultados pesquisados; esses revelaram grande desconhecimento a respeito de como garantir os direitos dos sujeitos de pesquisa, por conta da quase ausência e/ou do inadequado funcionamento dos comitês de ética de pesquisa.

Palavras-chave: sujeitos de pesquisa, comité de ética, principios bioéticos, dignidade humana

Maryknoll Sisters of Saint Dominic, Bolivia. Becaria del Programa de Ética de la Investigación Biomédica y Psicosocial, Fogarty Internacional Center, Grant R25 TW006056, 2007.

Correspondencia: ezwareva2004@yahoo.com 


\section{Introducción}

En Bolivia los problemas relacionados con la no importancia de la revisión y la deficiencia ética de los protocolos se centran en la escasez de los comités de ética de la investigación. Por otra parte, además de la inadecuada educación bioética de los miembros de estos comités, existe un desconocimiento de la bioética como materia esencial en la formación profesional del investigador.

En Bolivia los investigadores deberían considerar la relevancia de las siguientes preguntas: ¿qué se investiga? ¿Cómo se investiga? ¿Cómo se trata al sujeto de la investigación? La instancia que debe facilitar la reflexión es el Comité de Ética de Investigación, cuya tarea fundamental es brindar protección a las personas objeto de experimentación. Ezekiel Emanuel reduce los requisitos que hacen que la investigación sea ética al valor, validez científica, selección equitativa de los sujetos, proporción favorable de riesgo-beneficio, evaluación independiente, consentimiento informado y respeto a los sujetos inscritos(1). Por lo visto, estos aspectos no han sido considerados en la elaboración, presentación y ejecución de los proyectos en Bolivia. La falta de medidas de protección a los sujetos hace peligrar su salud y sus vidas.

Una reflexión auténtica se fundamenta en los principios bioéticos de autonomía, beneficencia, no maleficencia y justicia. Entre la ciencia y la bioética debe existir una relación dialógica, es decir, acción que se acompaña por reflexión.

Nuestro estudio -enfocado en los comités de ética de investigación biomédica y psicosocial en seres humanos-fue un intento por acercarnos a la situación actual de la bioética en Bolivia.

\section{La investigación en Bolivia}

Como miembro de la comunidad mundial, Bolivia se ha beneficiado con los avances de la biotecnología, producto de la investigación tecnocientífica. Nuestra hipótesis afirma que no hay suficientes instancias para proteger a la persona humana como sujeto de la investigación biomédica en este país y que la investigación en seres humanos se practica sin un control riguroso.

Las poblaciones vulnerables necesitan que los comités les brinden las condiciones de protección que no limiten su capacidad y libertad de consentimiento. Es preciso preguntarse qué leyes regulan la investigación en seres humanos. Se espera que el investigador actúe responsablemente y proteja a los sujetos de la investigación.

\section{Legislación boliviana}

La nueva Constitución Política del Estado, promulgada el 7 de febrero de 2009, establece que: "Es responsabilidad del Estado promover y garantizar el respeto, uso, investigación y práctica de la medicina tradicional, rescatando los conocimientos y prácticas ancestrales desde el pensamiento y valores de todas las naciones y pueblos indígenas originarios campesinos (Art. 42.I). (...) Ninguna persona será sometida a intervención quirúrgica, examen médico o de laboratorio sin su consentimiento o el de terceros legalmente autorizados, salvo peligro inminente de su vida (Art. 44.I). (...) Ninguna persona será sometida a experimentos científicos sin su consentimiento (Art. 44.II)" (2).

El Código de Ética y Deontología Médica de 2004 (artículo 103) menciona la necesidad de que los protocolos sean aprobados por un Comité de Investigación(3). Sin embargo, ¿qué se puede esperar si en la institución no existe un Comité que evalúe los protocolos de investigación? La velocidad del avance tecnocientífico desafía la reflexión bioética, la que debe incrementar su influencia en el ámbito de la investigación con seres humanos. Según Diego Gracia, resulta imposible "compaginar ciencia y vida evitando que haya conflictos", pero sí promover una "visión responsable", lo que se puede interpretar como tener un diálogo fructífero que ayude a mejorar la vida del hombre(4).

\section{La realidad sociocultural y la investigación}

¿Cómo se puede hablar de protección de la persona humana, sujeto de la investigación, sin considerar el ambiente en que vive? En Bolivia la pobreza y la violencia degradan el valor de la vida. Sin embargo, la tarea de la bioética es educar para la paz, para defender el derecho a la vida y promover el progreso. Presentar un proyecto de investigación que ofrezca como recompensa un poco de comida o un poco de dinero es suficiente para atraer la participación de gente pobre. No obstante, los comités de ética pueden identificar las poblaciones vulnerables y velar por su protección y bienestar y el Estado crear leyes que concuerden con las pautas internacionales.

Aunque la interdisciplinariedad de la bioética hace que sea posible incorporarla en cualquier campo de 
estudios, en Bolivia no se la reconoce como materia importante y esencial en el campo académico. La calidad de la bioética demanda un espacio único dentro de los programas de formación en investigación con seres humanos.

Para avanzar en la solución de los problemas de formación bioética era necesario estudiar la situación actual sobre el funcionamiento de los comités de ética en Bolivia y luego proponer la elaboración de programas de educación permanente en esta área. En el estudio, cuyos resultados aquí se publican, las preguntas fundamentales se enfocaron sobre la existencia de los comités en Bolivia, la educación de los miembros y la manera de proteger a los sujetos de la investigación. Según nuestro juicio, el estudio de los comités de ética de la investigación refleja el estado de la bioética en Bolivia, porque pregunta sobre el nivel de preparación de los miembros y los principios básicos que se deben seguir en la evaluación de protocolos y ensayos clínicos. El universo del estudio se limitó a las ciudades de La Paz, Cochabamba y Santa Cruz.

\section{Métodos}

Primero, se elaboró un cuestionario dirigido a los actores principales de instituciones educativas en salud e investigación. La pregunta de fondo fue: ¡cuál es la situación de la bioética en Bolivia y cómo se está protegiendo a los sujetos de investigación?

El instrumento se divide en tres partes: tenencia de comités, composición, conformación y funcionamiento de los comités y medida de los conocimientos sobre la investigación y la manera de proteger al sujeto humano.

Veintitrés representantes de instituciones participaron en el estudio. Se solicitó el consentimiento escrito de los participantes antes de aplicar el cuestionario. Al concluir el estudio, se analizaron los datos y se los separó en categorías.

\section{Resultados}

Después de categorizar las preguntas relacionadas, se analizaron los resultados tomando en cuenta cada pregunta y sus indicadores. Para asegurar la protección de la identidad de cada institución, se asignó un número a cada cuestionario.

Los resultados demostraron incertidumbre en cuanto a la definición de los comités de ética de la investigación.
Algunas instituciones indicaron que realizan investigación sin la presencia de una entidad evaluadora, por lo cual se esperaba que usaran la prestación de servicios de quienes sí tenían un comité de ética de la investigación, hecho que no fue así.

\section{Los comités de ética de la investigación}

El promedio de miembros que conforman el comité fue de siete, que se reúnen por lo menos una vez al mes. Pocos comités informan de la presencia de miembros legos durante las reuniones. No se define la forma de tomar decisiones, tampoco es claro qué cargos tienen los miembros. No se ha entendido el término "conflicto de interés" y cómo resolver los problemas que este problema suscita durante las sesiones.

La mayoría de los comités fueron establecidos entre 2003 y 2008. Sobre la figura legal que los crea, tres instituciones informaron que fue por resolución ministerial y el resto mencionó resoluciones institucionales o algún manual de la organización.

En cuanto a las actas, se observan discrepancias entre las instituciones que reportaron tener comités (un total de trece) y los que disponen de actas de las reuniones.

La mayoría de las instituciones (diecisiete) no informa sobre los protocolos evaluados, a causa de la falta de registros. Algunos participantes no tenían información alguna sobre los trabajos evaluados, de los cuales se excluyeron los retrospectivos, que se consideran trabajos científicos que involucran a sujetos humanos.

Algunos representantes mencionaron dificultades relacionadas con la regularidad de las reuniones, asistencia de los miembros y múltiple conformación del Comité; otros, la escasa disponibilidad de tiempo para evaluar proyectos y serias dificultades de financiamiento para el funcionamiento del Comité.

El estudio indica que los miembros son en su mayoría profesionales y con muchas responsabilidades. Los problemas indicados se vinculan estrechamente con las mismas funciones que se van realizando y que no permiten dedicar más tiempo a la evaluación de protocolos ni a las reuniones. También se percibe incertidumbre en cuanto a la ejecución de funciones como comité evaluador, problema que sólo se podrá solucionar con una buena formación de los miembros. 


\section{La formación bioética de los miembros}

Los miembros de los comités adquirieron su formación en bioética de varios modos: asistencia a congresos de bioética, seminarios, Internet, entre otros. Otras instituciones indicaron que no tenían estudios específicos en el área de la bioética. Entre las instituciones que no informaron y las que dijeron no tener formación alguna suman el $61 \%$.

Formación Bioética de los miembros

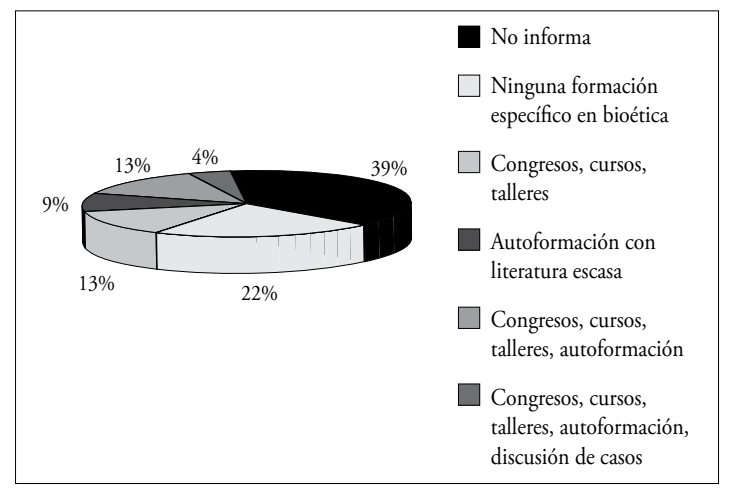

Es importante indicar que se han realizado cuatro congresos bolivianos de Bioética después del nacimiento del "Comité Impulsor de Ética y Bioética, el 7 de septiembre de 2000. El Primer Congreso Boliviano de Bioética tuvo lugar en 2001, durante la realización se formaron los comités departamentales de bioética..." (5). El segundo Congreso se realizó en 2003; el tercero en 2005 y el cuarto en marzo de 2008.

Los problemas expresados por las instituciones reflejan la necesidad de que los directores reconozcan la importancia de la participación de los miembros de comités en estos encuentros. El estudio revela mucho interés en tener vínculos con otras instituciones para formar grupos cooperativos institucionales en ensayos clínicos y nutrir las propias experiencias. La conformación de vínculos puede ser un instrumento para una colaboración estrecha, que quiebre la competividad y la indiferencia que impide el avance de la bioética en el país.

\section{Diferencias entre los comités}

Los resultados muestran un grado de confusión respecto de la diferencia entre un comité de ética de la investigación y otros comités. Es necesario educar para que estos conceptos se clarifiquen. El trabajo de la evaluación de los protocolos es una tarea exigente; por lo tanto, es importante no saturar a los comités de ética de la investigación con otras tareas que correspondan a otra clase de comités. Confundir su rol puede repercutir en la calidad de la evaluación y el posterior seguimiento de los proyectos.

\section{El reglamento sobre la investigación}

Once entidades afirmaron conocer la existencia de un reglamento nacional sobre la investigación en seres humanos, pero cuatro de ellas mencionan "algún" reglamento. Siete dijeron que no existe un reglamento, tres lo desconocen y dos no respondieron. La mayoría (doce instituciones) está de acuerdo con la necesidad de tener un reglamento, mientras once no respondieron a la pregunta. Todos los participantes consideraron necesario un reglamento sobre la investigación en seres humanos en Bolivia.

El actual Código de Salud se refiere a la protección del sujeto de la investigación en el Decreto Ley No 15.629 de 18 de julio de 1978 (Artículos 147 y 148)(3). Igualmente, el Código de Ética Médica del Colegio Médico de Bolivia dedica unos artículos a la investigación biomédica y reconoce su importancia y la necesidad de seguir normas y pautas nacionales e internacionales sobre la investigación en seres humanos(6).

Para una mayor relevancia, el reglamento necesita una instancia oficial que impulse su establecimiento y asegure su observancia. Aunque la mayoría no indicó una instancia impulsora, cinco mencionaron al Ministerio de Salud, además de otras sugerencias.

\section{La revisión ética}

Once representantes de las instituciones expresaron que la revisión ética de los protocolos de investigación no interfiere con el proceso de la investigación. Seis no respondieron, cinco afirmaron que la revisión ética sí interfiere y una que la revisión ética interfiere y no interfiere con el proceso de la investigación. Para una mayoría, la revisión ética es necesaria y obligatoria (de veintitrés instituciones), dos consideran que no es necesaria ni obligatoria. Sólo una institución no respondió a esta pregunta.

Algunos piensan que el mismo investigador debe evaluar sus propios proyectos, pero el peligro mayor es el de la omisión de sus propios errores éticos. Seis no respondieron y siete estaban de acuerdo con que lo realice el mismo investigador. Nueve no estaban de acuerdo con la autoevaluación y uno optó por los dos medios. 
Un 83\% los encuestados está de acuerdo con que la evaluación ética debe continuar después de la aprobación y un $17 \%$ no contestó. El $78 \%$ no indicó la modalidad del análisis ético, mientras que los otros indicaron que debía ser mediante reglamentos (9\%), documentos nacionales e internacionales (9\%), y que deben realizarla los comités de ética de la investigación (4\%).

\section{Conclusiones}

Los resultados del estudio revelan una carencia en el conocimiento de cómo proteger a la persona humana en la investigación. Existe una tendencia negativa que indica la casi ausencia o el inadecuado funcionamiento de los comités. No se diferencian los comités de ética de la investigación de otros comités.

La falta de financiamiento y de apoyo a los comités limita la participación de los miembros, los cuales deben reunirse en su tiempo libre y buscar la manera de cubrir los gastos implicados en su organización y funcionamiento. Además de una insuficiencia en la formación bioética de los miembros, no se conoce la existencia de normas que regulen la investigación en seres humanos en Bolivia.

\section{Recomendaciones}

Tomando en cuenta los resultados de este estudio, se debe iniciar un diálogo interinstitucional que ayude a buscar una manera eficaz y seria de implementar comités de ética de la investigación, tomando como modelos los comités existentes. Según uno de los participantes en el estudio: "En Bolivia será necesa- rio impulsar niveles académicos formales y, hasta el presente, ninguna universidad ha mostrado interés en hacerlo", afirmación que se debe tomar en serio y buscar respuestas concretas.

Es necesario avanzar en el conocimiento de las normas y guías internacionales sobre la investigación en seres humanos, a partir de las cuales se elaboren normas nacionales que puedan guiar la investigación en Bolivia. Las instancias indicadas para la tarea son: el Comité Impulsor de la educación en Bioética, el Ministerio de Salud y el Gobierno Nacional.

Es indispensable contar con un organismo que pueda acreditar a los comités y asegurar su conformidad con los reglamentos internacionales y nacionales, además de tener un reglamento nacional sobre la protección de sujetos de la investigación biomédica y psicosocial. El Ministerio de Salud debe preocuparse por difundir la existencia de reglamentos y leyes referentes al tema.

Se recomienda una mayor colaboración interinstitucional y generosidad al compartir conocimientos y materiales. Considerando la importancia de la educación en bioética, es menester que las autoridades asignen fondos para el proyecto y no esperar que las personas aporten sus propios recursos para este fin.

\section{Agradecimientos}

Al Centro Interdisciplinario de Estudios en Bioética (CIEB) de la Universidad de Chile (Programa de Ética de la Investigación Biomédica y Psicosocial, Fogarty Internacional Center, Grant R25 TW006056, 2007) y a la Congregación de las Hermanas de Maryknoll, entidades que desinteresadamente apoyaron el desarrollo del estudio hasta su término.

\section{Referencias}

1. Lolas F, Quezada A. Pautas éticas de investigación en sujetos humanos: nuevas perspectivas. Santiago de Chile: Programa Regional de Bioética OPS/OMS; 2003: 85-90.

2. Constitución Politica del Estado. Promulgada el 7 de febrero de 2009. La Paz: Librería Editorial MULTI; 2009.

3. Código de Salud. Decreto de Ley No 15.629 de 18 de julio de 1978. U.P.S. La Paz (Bolivia); 2005.

4. Gracia D. Profesión médica, investigación y justicia sanitaria. En: Ética y vida, 4. Ed. Santa Fe de Bogotá: El Búho; 1998.

5. Luna Orosco J. La bioética en Bolivia: antecedentes y proyecciones. En: Pessini L, Barchifontaine CP, Lolas F. (coords.) Perspectivas de la Bioética en Iberoamérica. Santiago de Chile: Programa de Bioética OPS/OMS; 2007.

6. Estatuto Orgánico del Colegio Médico de Bolivia, 2004. Disponible en http://www.colegiomedicodebolivia.org.bo/ documentos/ESTATUTO_ORGANICO.pdf, último acceso en mayo 5, 2010.

Recibido: 17 de marzo de 2009

Aceptado: 23 de octubre de 2009 